\title{
DISEÑO Y MANUFACTURA DE UN PROTOTIPO DE ESCUDO FACIAL CON RESPIRADOR QUE EVITE EL CONTAGIO DEL COVID-19 (1. Etapa)
}

\author{
DESIGN AND MANUFACTURE OF PROTOTYPE OF FACIAL SHIELD WITH A \\ RESPIRATOR TO AVOID THE SPREAD OF COVID-19 (1 ${ }^{\text {ST }}$ PHASE)
}

\author{
Diego Machuca V.M. ., Juan Carlos Gonzalez L.* , \\ Carlos Merino M. ${ }^{* * *}$ y María Teresa Méndez L.***1
}

RECEPCIÓN: 05 DE NOVIEMBRE DE 2020

ACEPTACIÓN: 11 DE DICIEMBRE DE 2020

\section{RESUMEN}

A lo largo del año 2020, el mundo ha sufrido los estragos de una pandemia de enfermedad por coronavirus, iniciada en 2019 (COVID-19) y causada por el coronavirus 2 del síndrome respiratorio agudo grave (SARS-CoV-2). En el Perú, el aislamiento social obligatorio ha generado grandes pérdidas económicas, que se han traducido en pérdida de empleos. La falta de adecuados equipos de protección sanitaria que brinden condiciones de aislamiento físico seguro dificulta la posibilidad para regresar a un "estado de normalidad" del aparato productivo. Basados en esta problemática, el Centro de Estudios para Comunidades Saludables de la Universidad Ricardo Palma, se planteó diseñar y manufacturar un Prototipo de Escudo Facial con Respirador que evite el contagio del COVID- 19. Basados en información previamente publicada y en tecnologías disponibles en nuestro país, se planteó una solución práctica para evitar el contagio del virus. El escudo facial cuenta con un sistema electrónicodigital con sensores de proximidad (HC-SR04) para detectar la aproximación dentro del radio de 1.5 metros, y sensores de temperatura MLX90614 para detectar si el usuario presenta temperatura superior a los $37.5^{\circ} \mathrm{C}$. Para visualizar los datos de temperatura del usuario, el Escudo Facial cuenta con un Módulo OLED. Toda la información recopilada por los sensores es identificada por el microcontrolador NodeMCU ESP8266, y el Arduino Nano V3 como controlador encargado de la recepción de los sensores de proximidad, comunicados de manera Serial ( $T x / R x)$.

Palabras clave: Escudo facial, COVID-19

\begin{abstract}
Throughout 2020, the world has suffered the ravages of a coronavirus disease pandemic, started in 2019 (COVID-19) and caused by the severe acute respiratory syndrome coronavirus 2 (SARS-CoV-2). In Peru, compulsory social isolation has generated great economic losses, which have resulted in job losses. The lack of adequate sanitary protection equipment that provides safe physical isolation conditions makes it difficult to return to a "normal state" of the productive apparatus. Based on this problem, the Center for Studies for Healthy Communities of the Ricardo Palma University, set out to design and manufacture a Prototype of a Face Shield with Respirator that prevents the spread of COVID-19. Based on previously published information and technologies available in our country, a practical solution was proposed to avoid the spread of the virus. The face shield has an electronicdigital system with proximity sensors (HC-SR04) to detect the approach within a radius of 1.5 meters, and MLX90614 temperature sensors to detect if the user has a temperature higher than $37.5^{\circ} \mathrm{C}$. To view the user's temperature data, the Face Shield has an OLED Module. All the information collected by the sensors is identified by the NodeMCU ESP8266 microcontroller, and the Arduino Nano V3 as the controller in charge of receiving the proximity sensors, communicated in a Serial ( $T x / R x)$ manner.
\end{abstract}

Keywords: Facial Shield, COVID-19

\footnotetext{
* $\quad$ Arquitecto, investigador principal del Proyecto. ${ }^{* *}$ Ingeniero, co-investigador del Proyecto. ${ }^{* * *}$ PhD., asesor del Proyecto. **** Dra. arquitecta, coordinadora del Proyecto.

1 Centro de Estudios para Comunidades Saludables - Universidad Ricardo Palma (CECOS-BRGURP). Email de contacto: maria.mendez@urp.edu.pe; cecos@urp.edu.pe
} 
Hasta el mes de diciembre del presente 2020, el mundo está sufriendo los estragos de una pandemia originada por la enfermedad del coronavirus iniciada en 2019 (COVID-19) [1] y causada por el coronavirus 2 del síndrome respiratorio agudo grave (SARS-CoV-2) [2]. La OMS la reconoció como una pandemia global el 11 de marzo de 2020 . Al 13 de abril del mismo año se reportaron más de 1.87 millones de casos de COVID-19 en más de doscientos países y territorios, lo que ha generado más de 116000 muertes y más de 441000 casos de recuperación. Merino, biólogo genetista molecular, explica que:

...se han hecho varias investigaciones para determinar el origen del virus que causa COVID-19. Se ha determinado que es un virus de origen natural [...] una quimera o híbrido de un virus de murciélago y un virus de otro animal. Este proceso de hibridación ocurre cuando ambos virus se encuentran en el mismo organismo hospedero. Los virus mutan con mucha facilidad y por eso son tan difíciles de combatir por el sistema inmune humano [...] análisis virológicos detallados demuestran que el virus se reproduce activamente en los tejidos de las vías aéreas superiores, la garganta y los pulmones. De hecho, la liberación del virus desde la faringe es muy alta durante la primera semana de síntomas y se ha encontrado virus infeccioso en abundancia en muestras derivadas de la garganta y los pulmones. También ha quedado demostrado que la liberación de ARN (material genético) de virus en esputo ocurre incluso después que terminan los síntomas. Aunque se ha hallado una alta concentración de ARN del virus en muestras de heces, no se ha hallado virus infecciosos en las mismas. Tampoco se ha podido extraer virus infeccioso de la sangre o la orina. [3].

Esta pandemia, en la población del Perú, está generando dos problemas fundamentales:

a. De salubridad, el más importante, puesto que, si no se guardan las medidas necesarias, el nivel de contagio en la población se convierte en exponencial, lo que provoca un nivel de mortandad incontrolable. Esto es causado por la permanencia del virus en el aire.

Por este motivo, y con la finalidad de hallar formas de controlar este problema, se realizaron estudios para estimar la transmisión del virus a través del aire, utilizando simulaciones en espacios cerrados. El Instituto de Tecnología de Kioto ha utilizado cámaras de alta sensibilidad para captar las micropartículas que quedan en el aire en suspensión cuando una persona habla, tose o estornuda [4]. Por eso, es de suma importancia contar con equipo de protección personal para usar en público si queremos evitar el contagio de esta enfermedad.

A fin de proteger a los individuos de los contagios, el gobierno del Perú ha dado una serie de recomendaciones. Lamentablemente, el incumplimiento de las normas de distanciamiento social dictadas por las autoridades de salud, causa, hasta la fecha, el incremento de casos de contagio y requiere de acciones especializadas para limitar el contagio entre personas que no cumplen las normas y directivas oficiales. Según expertos en conducta social:

...el ser humano es un ser social por naturaleza [...]. Nos necesitamos los unos a los otros para sobrevivir. Somos más que un conjunto de células. La cuarentena es antinatural es ir contra nuestra propia naturaleza. De ahí la necesidad de la gente de salir, de acercarse a otro... [5]

A partir de lo planteado, se explica por qué es tan difícil acatar la cuarentena y también por qué, cuando la respetamos, afecta nuestra salud mental y emocional.

Este comportamiento ha causado gran preocupación en el entorno de las autoridades de salud. De hecho, el Ministro de Salud [6] declaró que "Un grupo va a morir en el hospital; otro, en la calle, en albergues o en sus casas. Para esto se creará un comando humanitario de levantamiento de cadáveres". La situación es aún más complicada en las zonas de más densa población como 
lo es nuestra capital, donde el problema del contagio se ve exacerbado de la misma manera que en las grandes metrópolis como Nueva York, Milán y Madrid. Esto se debe a que, en las grandes ciudades, la densidad poblacional es mayor, lo que dificulta el distanciamiento social.

b. Económico, generado por el aislamiento social obligatorio, el mismo que ha provocado una gran pérdida económica en el sector empresarial al prácticamente paralizarlo. Esto se ha traducido como pérdida de empleo entre la población peruana en general, pese a las medidas económicas adoptada por el gobierno peruano. La imposibilidad de reabrir los negocios y mantener la distancia social para prevenir el contagio del COVID-19 ha generado un impacto global económico en el plan de los gobiernos para afrontar la cuarentena.

Se observa así que la falta de acceso a adecuados equipos de protección personal para la población en general ha incrementado la dificultad y riesgo para regresar a un estado de normalidad del aparato productivo, puesto que no se cuentan con condiciones de aislamiento físico seguro. En consecuencia, para insertarse de manera segura al aparato productivo es necesario contar con un equipo de protección personal que garantice a la población evitar el contagio del COVID-19 por personas con las que interactúen durante el cumplimiento de sus funciones laborales.

Analizando esta problemática, el Centro de Estudios para Comunidades Saludables de la Universidad Ricardo Palma, se plantea diseñar y manufacturar un Prototipo de Escudo Facial con Respirador que evite el contagio del COVID- 19. Este tiene los siguientes objetivos específicos:

a) Diseñar una máscara que selle el contorno del rostro del usuario, adaptándose a las tallas de diferentes personas, lo que ha producido un aislamiento total del rostro con el objetivo de minimizar el riesgo de contagio:

b) Analizar comparativamente el diseño de la máscara para que su manufactura solamente emplee insumos existentes en el mercado nacional, y de bajo costo, al mismo tiempo que cumpla con el estándar de filtración necesario para evitar el paso de partículas virales; y

c) Diseñar un circuito electrónico integrado a la máscara que permita mantener registradas las condiciones de temperatura del usuario, la distancia social de aislamiento y la posibilidad de respirar a través de filtros protectores y un sistema de ventilación que evite el empañamiento del visor.

Todo esto es posible adaptando tecnología de libre acceso y bajo costo existentes en el país para el diseño, manufactura y testeo de equipos de protección personal, con el cumplimiento de los protocolos de seguridad requeridos para evitar el contagio del virus.

El estudio está basado en el método hipotético deductivo, partiendo de una hipótesis o explicación inicial, para luego obtener conclusiones particulares de ella, que serán a su vez comprobadas experimentalmente. Basados en información previamente publicada y en tecnologías disponibles en nuestro país, proponemos implementar una solución práctica para evitar el contagio del virus causante del COVID-19.

En el diseño del prototipo, se emplearán herramientas basadas en teorías de Arquitectura Digital, como es el Diseńo Paramétrico para el modelado del objeto resultante, que, a diferencia de un método de diseño tradicional, permite representar en 3 dimensiones los patrones o comportamientos según las diferentes variables. Las herramientas empleadas para este tipo de diseño son softwares especializados como la interfase Grasshopper, editor gráfico de algoritmos que permite a los diseñadores crear generadores de forma, y la metodología CAM, que permite transferir la información de la computadora a una máquina para la construcción del modelo resultante, como son la impresión 3D y el Corte Láser. 
La Impresión 3D o 3D Printing permite realizar impresiones de las variables del diseño tridimensional, a partir del modelo 3D hecho en el computador. El Corte L.A.S.E.R. (CNC), o corte controlado por computador, es una herramienta de precisión que permite el corte de las piezas con alta precisión a una altísima velocidad, con un mínimo de pérdida de material y con distorsiones menores a un milímetro. Adicionalmente, se utilizará el Blender, un software que se emplea como parte del proceso de diseńo y modelamiento de rostro para recrear de una manera exacta sus dimensiones y poder usar esas medidas como punto de partida para el diseńo de la máscara.

El diseño electrónico emplea el Arduino como una plataforma para la toma de información del entorno a través de sensores y para controlar aquello que le rodea. El microcontrolador en la placa Arduino cuenta con su propio lenguaje de programación (basado en Wiring) y entorno de desarrollo (basado en Processing). Además del diseño del Escudo Facial, se tiene un sistema electrónico-digital que cuenta con sensores de proximidad (HC-SR04) para detectar la presencia de usuarios dentro de un mismo espacio (aproximación dentro del radio de 1.5 metros), así como sensores de temperatura MLX90614, para detectar si el usuario presenta un cuadro de fiebre (temperatura superior a los $37.5^{\circ} \mathrm{C}$ ). Con la finalidad de visualizar los datos de temperatura del usuario, el Escudo Facial cuenta con un Módulo OLED. Adicionalmente, cuenta con un sensor de presión atmosférica y altura sobre el nivel del mar (BMP y DHT11) para poder realizar un control adicional sobre el sistema de ventilación considerado para el prototipo, el cual se encuentra en etapa de ensayo.

Toda la información recopilada por los sensores es identificada por el microcontrolador, siendo en este caso el NodeMCU ESP8266 el controlador Maestro (encargado de la recepción de los sensores de temperatura, Presión atmosférica y Microcontrolador Esclavo) y el Arduino Nano V3 como controlador Secundario (encargado de la recepción de los sensores de proximidad). Ambos controladores se comunican de manera Serial ( $\mathrm{T} x / \mathrm{Rx})$.

Para el control adicional se utiliza el Microcontrolador ESP-CAM 32 el cual tiene como agregado la cámara OV2640, la que permite realizar streaming de video e imágenes, las que pueden ocasionalmente, ser enviadas a un servidor local para su almacenamiento y visualización, posibilitando de esta manera mantener lo registrado por la cámara. El microcontrolador WeMos-Mini y Arduino UNO R3 se utilizan como dispositivos independientes que permiten adicionar funciones al Protector Facial.

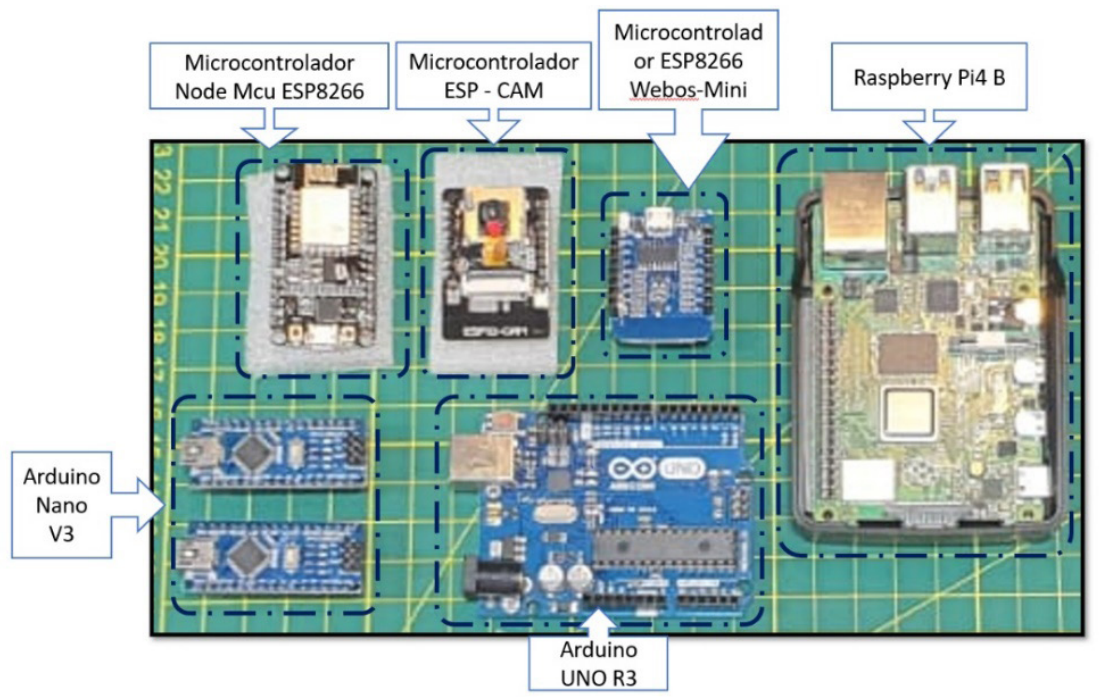

Figura 1 . 
El Raspberry Pi4 B, al ser una minicomputadora con un alto nivel de procesamiento, es empleado como un servidor web local, que se encuentra en desarrollo y en prueba de diagnóstico. Este servidor permite realizar el procesamiento de un número considerable de usuarios de los escudos faciales de manera individual, lo que permite mantener un registro constante de los diferentes usuarios dentro de un mismo grupo, tanto de su estado de aislamiento como condiciones de presión atmosférica, ubicación, y temperatura de usuario.

Con el propósito de mantener un registro de la ubicación de cada usuario agregado a un grupo en el servidor desarrollado en el Raspberry, el dispositivo cuenta con un Módulo de Ubicación GPS NEO-8 que, en un caso de urgencia de salud, permite ubicar de inmediato al usuario.

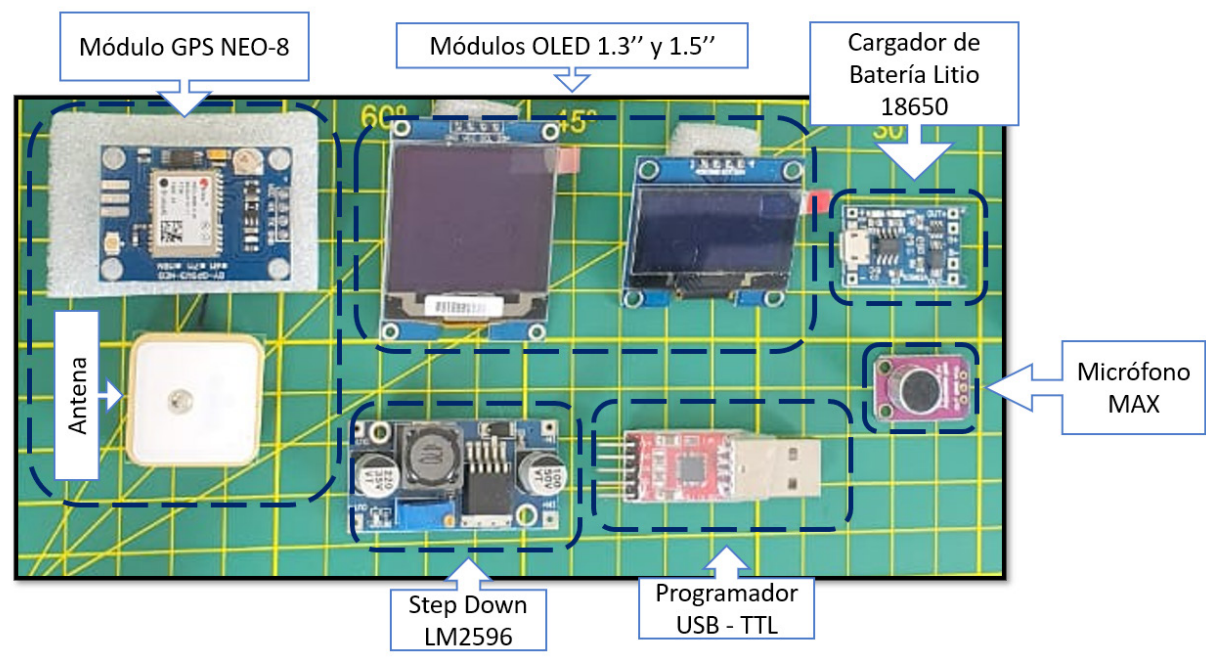

Figura 2 .

Para el sistema de alimentación se cuenta con un cargador de batería de litio 18650 que permite cargar las baterías y brindarle autonomía al prototipo. Las pruebas mostraron una duración de entre 4 y 5 horas de funcionamiento.

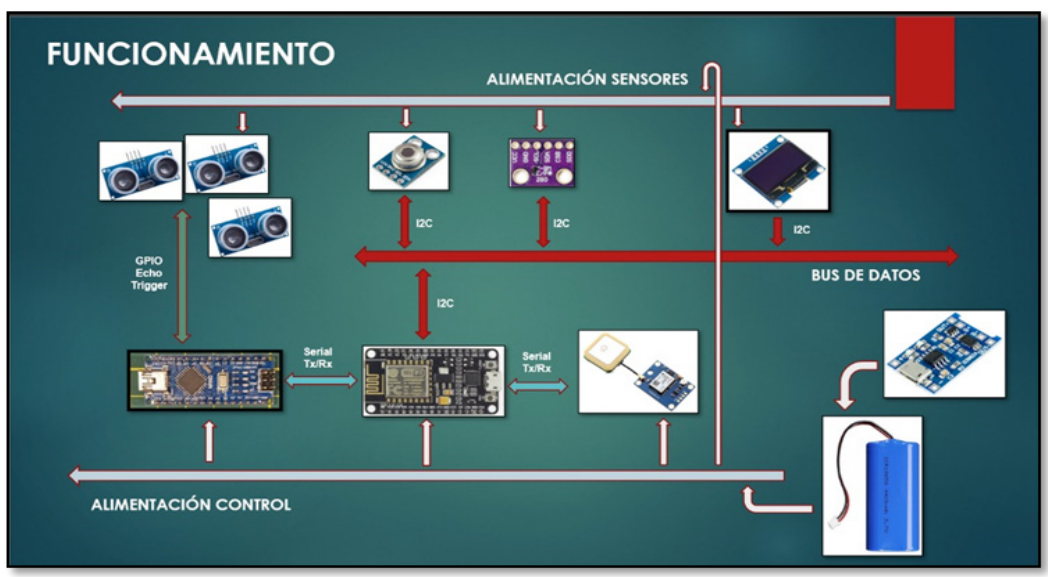

Figura 3. 


\section{RESULTADOS}

Escaneado 3D / Modelado Facial. Se realizaron pruebas con diferentes rostros. El modelado inicial se parametrizó para que las modificaciones en el diseño sean mucho más rápidas en su proceso. Asimismo, se modeló el lienzo escogido y se trabajó en el software Blender para su posterior traslado al Rhinoceros para continuar con el modelado de la máscara. Como se observa en el diseńo final de la máscara, esta se amolda al rostro de manera ergonómica al igual que las cintas de ajuste de 3 puntos para que no se resbale del rostro.

Optimización de la recolección de datos 3D. De acuerdo con los diferentes rostros de personas, se parametrizaron algunos elementos que pueden variar en el rostro como la zona de los pómulos, el ancho de la frente, el tamańo de la nariz y la distancia entre la frente y la mandíbula. Toda esta información es necesaria, puesto que de estos datos dependerá la funcionalidad del diseño, incluso se considera que algunos usuarios podrían portar lentes.

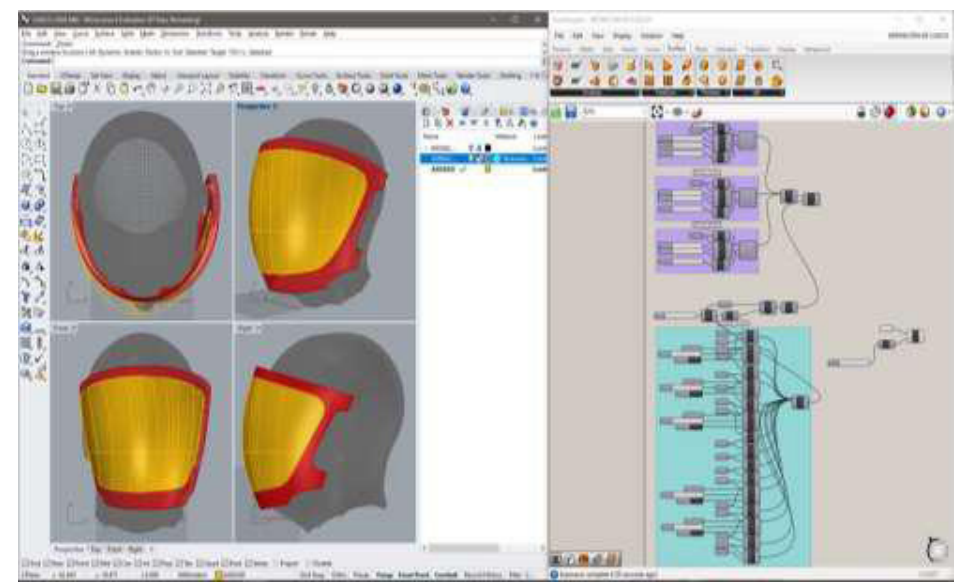

Figura 4 .

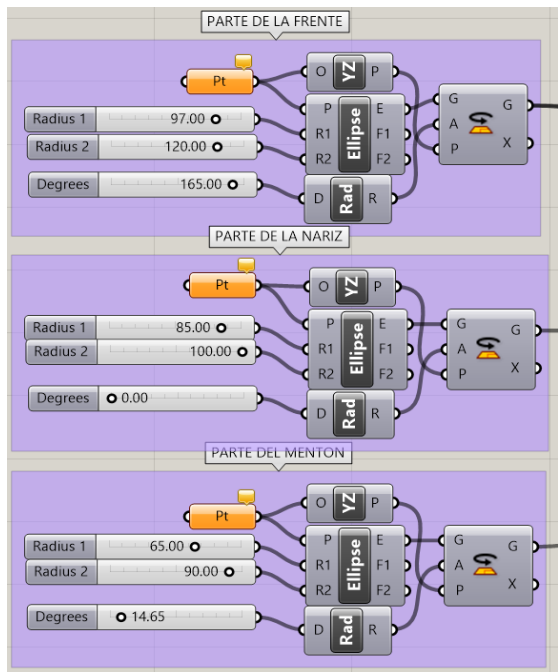

Figura 5. 
Diseño funcional. Se ha concluido con la primera parte del proyecto consistente en el diseño de la máscara con la incrustación de los sensores de proximidad, las baterías y el sensor de temperatura. El ensamblado se ha realizado de manera análoga al casco, y se han desarrollado algunas modificaciones (parches) en los cortes análogos para, posteriormente, realizar el pulido y pintado del prototipo.

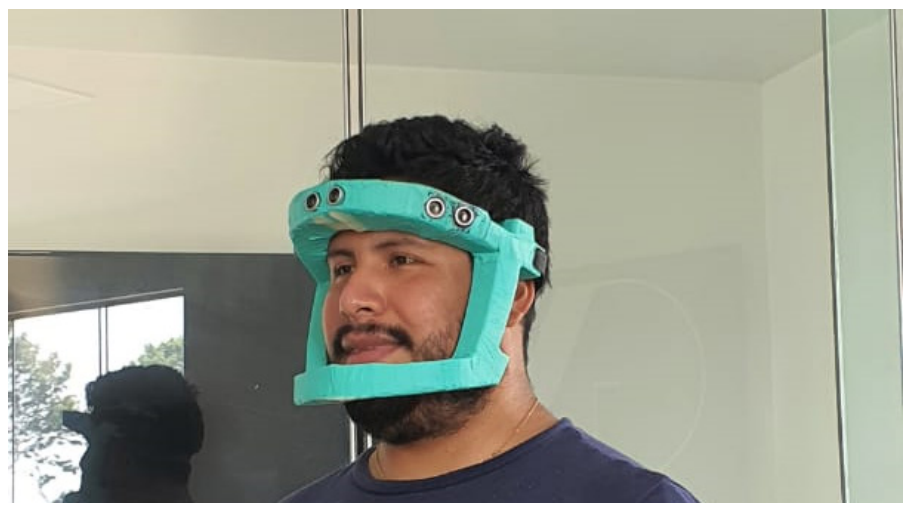

Figura 6.

Queda pendiente la resolución de la fase funcional. El modelo fue impreso en 3D con un material plástico denominado PLA, pero en la práctica se presentaron algunas dificultades de trabajo, por lo que se está analizando diferentes opciones, como variables del material a utilizar, para su fabricación. A la fecha, el equipo de investigadores se encuentra realizando pruebas con otros materiales de fácil moldeado análogo (manual) para sellar algunas porosidades que deja la impresora 3D durante la construcción del modelo. Asimismo, se está verificando la duración de la impresión, y afinando la cantidad de material que se utilizará en las diferentes etapas.
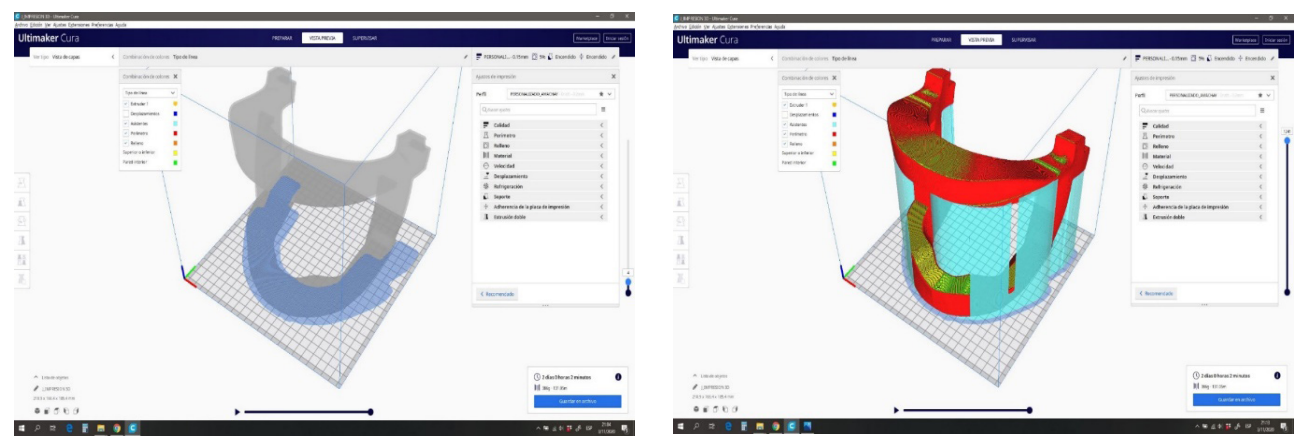

Figura 7.

\section{CONCLUSIONES Y RECOMENDACIONES}

- Se realizaron algunas modificaciones (parches) en los cortes análogos para el posterior pulido y pintado del prototipo.

- Respecto al modelo 3D, el diseño inicial ha sido modificado a fin de que la parte electrónica se encuentre incluida dentro del casco. 
- El tiempo de vida útil por medio de las baterías de litio es de un día completo si no tiene carga conectada. El tiempo de vida conectada a todos los sensores, microcontroladores y dispositivos es de 3 a 5 horas.

- Se recomienda utilizar el módulo Node MCU - ESP8266, debido a que presenta mayor capacidad de memoria para un trabajo óptimo con los sensores y dispositivos.

- Es preferible utilizar la información obtenida por el módulo GPS para brindarle al usuario información necesaria y útil, como fecha, hora, y datos agregados en el registro brindado por los GPS.

- El uso de sensores ultrasónicos debe presentar una mayor protección contra el polvo y el agua. Por tal motivo, se ha pensado iniciar pruebas con los sensores JSN-SR04T, los cuales presentan una mayor proyección ante la lluvia y el polvo.

- Queda pendiente de trabajar el acondicionamiento de las placas y sensores dentro del modelo, por lo que se recomienda una segunda etapa que comprenda ensayos de funcionamiento y producción respectivas, antes de su introducción al mercado.

- Es importante realizar pruebas de ensayo de funcionamiento con personas, para verificar y solucionar problemas de respiración y empañamiento de la mica, así como la colocación de otros accesorios electrónicos que se pudieran requerir.

\section{REFERENCIAS}

[1] "Pandemia de Covid-19", Wikipedia, 2020. [En línea]. Disponible en: https:/es.wikipedia.org/ wiki/Pandemia_de_enfermedad_por_coronavirus_de_2019-2020_[Accedido: 28-dic-2020].

[2] "Síndrome respiratorio agudo grave", Wikipedia, 2020. [En línea]. Disponible en: https:// es.wikipedia.org/wiki/S\%C3\%ADndrome_respiratorio_agudo_grave [Accedido: 28-dic-2020].

[3] C. Merino (2020)

[4] “Cuánto y cómo permanece el coronavirus en el aire?: el video que muestra el riesgo de una simple tos”, La Sexta, 2020. [En línea]. Disponible en: https://www.lasexta.com/programas/al-rojovivo/noticias/cuanto-y-como-permanece-el-coronavirus-en-el-aire-el-estudio-que-advierte-de-ladistancia-a-mantener-al-hacer-deporte_202004105e907141a8aed2000124f5a5.html [Accedido: 28-dic-2020]

[5] E. Arrieta, "El hombre es un ser social por naturaleza", Cultura genial, 2020. [En línea]. Disponible en: https://www.culturagenial.com/es/el-hombre-es-un-ser-social-por-naturaleza/

[Accedido: 28-dic-2020].

[6] "Víctor Zamora: 'Un grupo va a morir en el hospital; otro, en la calle, en albergues o en sus casas”, Perú21, 2020. [En línea]. Disponible en: https://peru21.pe/peru/coronavirus-peru-ministro-de-salud-se-creara-un-comando-humanitario-de-levantamiento-de-cadaveres-covid-19-estado-de-emergencia-toque-de-queda-aislamiento-social-obligatorio-minsa-noticia/ [Accedido: 28-dic-2020] 